EPJ Web of Conferences 101, 02006 (2015)

DOI: 10.1051/epjconf/201510102006

(C) Owned by the authors, published by EDP Sciences, 2015

\title{
HD 97658 and its super-Earth
}

\author{
V. Van Grootel ${ }^{1, a}$, M. Gillon ${ }^{1}$, D. Valencia ${ }^{2}$, N. Madhusudhan ${ }^{3}$, D. Dragomir ${ }^{4,5}$, A.R. Howe ${ }^{6}$, and \\ A.S. Burrows ${ }^{6}$ \\ 1 Institut d'Astrophysique et de Géophysique, Université de Liège, Belgium \\ 2 Department of Physical and Environmental Sciences, University of Toronto, Canada \\ 3 Institute of Astronomy, University of Cambridge, UK \\ 4 Las Cumbres Observatory Global Telescope Network, USA \\ 5 Department of Physics, Broida Hall, UC Santa Barbara, USA \\ 6 Department of Astrophysical Sciences, Princeton University, USA
}

\begin{abstract}
Super-Earths transiting nearby bright stars are key objects that simultaneously allow for accurate measurements of both their mass and radius, providing essential constraints on their internal composition. We present the confirmation, based on Spitzer observations, that the super-Earth HD $97658 \mathrm{~b}$ transits its host star. HD 97658 is a lowmass $\left(M_{*}=0.77 \pm 0.05 M_{\odot}\right) \mathrm{K} 1$ dwarf, as determined from the Hipparcos parallax and stellar evolution modeling. To constrain the planet parameters, we carry out Bayesian global analyses of Keck-HIRES radial velocities, and MOST and Spitzer photometry. HD $97658 \mathrm{~b}$ is a massive $\left(M_{P}=7.55_{-0.79}^{+0.83} M_{\oplus}\right)$ and large $\left(R_{P}=2.247_{-0.095}^{+0.098} R_{\oplus}\right.$ at $\left.4.5 \mu \mathrm{m}\right)$ super-Earth. We investigate the possible internal compositions for HD 97658 b. Our results indicate a large rocky component, by at least $60 \%$ by mass, and very little $\mathrm{H}-\mathrm{He}$ components, at most $2 \%$ by mass. We also discuss how future asteroseismic observations can improve the knowledge of the HD 97658 system, in particular by constraining its age.
\end{abstract}

\section{Introducing HD 97658 and its super-Earth}

HD 97658 b was detected from radial velocity (RV) measurements with Keck-HIRES spectroscopy, from which a minimum mass $M_{\mathrm{P}} \sin i=8.2 \pm 1.2 M_{\oplus}$ and an orbital period $P=9.494 \pm 0.005$ days were derived [1]. MOST observations discovered transit events with an ephemeris consistent with that predicted from RV measurements [2]. This transiting nature allowed for measurement of the planetary radius and true mass, making HD 97658 the second brightest $(V=7.7, K=5.7)$ star found to be transited by a super-Earth. This fact motivated us to include HD $97658 \mathrm{~b}$ in our Spitzer program to search for and observe transits of low-mass RV-detected planets [3], and HD $97658 \mathrm{~b}$ was observed on 2013 Aug 10 with the Spitzer IRAC camera at $4.5 \mu$ m during 6 hours corresponding to a transit window as computed from the MOST transit ephemeris.

Ultimately, the precision for planetary parameters is related to the precision achieved for the stellar parameters, since planetary masses and radii as determined by transit and RV measurements cannot be determined independently from the properties of their host stars. The atmospheric parameters of HD 97658 are [1]: $T_{\text {eff }}=5170 \pm 44 \mathrm{~K}, \log g=4.63 \pm 0.06$, and $[\mathrm{Fe} / \mathrm{H}]=-0.23 \pm 0.03$. A Hipparcos parallax measurement of HD 97658 is available, from which we derived the stellar luminosity $L_{*} / L_{\odot}=$ $0.355 \pm 0.018$ (from distance, observed and computed absolute magnitudes. The full calculation can be found in [4]). Combining the stellar luminosity $L_{*} / L_{\odot}=0.355 \pm 0.018$ with the effective temperature of [1] results in a stellar radius $R_{*} / R_{\odot}=0.74 \pm 0.03$. In order to derive the stellar mass and age, we used the effective temperature, metallicity and luminosity with their respective errors as inputs

a e-mail: valerie.vangrootel@ulg.ac.be

This is an Open Access article distributed under the terms of the Creative Commons Attribution License 4.0, which permits unrestricted use, distribution, and reproduction in any medium, provided the original work is properly cited. 


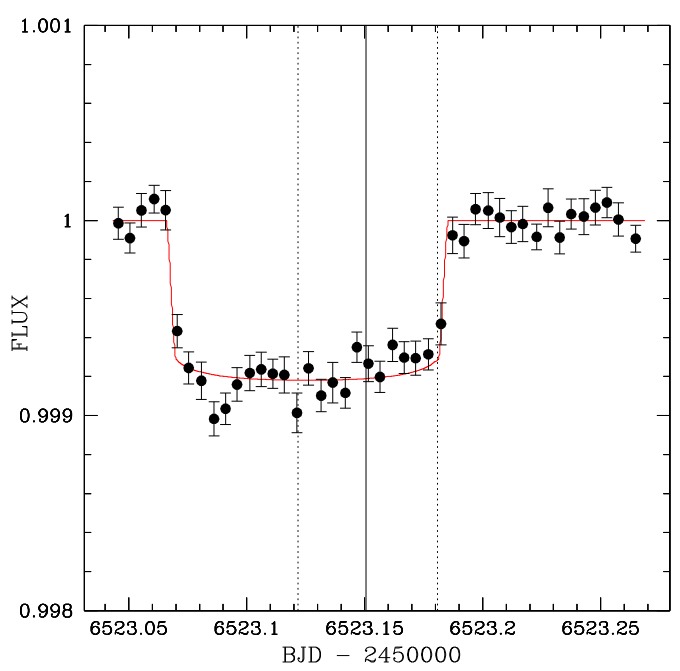

Fig. 1. HD 97658 Spitzer IRAC light curve corrected from the Spitzer instrumental effects (see [4]) and binned to intervals of five minutes, with the best-fit transit model overimposed. The vertical solid and dashed lines are the propagated mid-transit time of [2] with its $1-\sigma$ errors.

for stellar evolution modeling with the Liege evolution code CLES [5]. We obtained a stellar mass $M_{*}=0.77 \pm 0.05 M_{\odot}$, but no useful constraint on the stellar age was obtained [4].

\section{Global MCMC analyses of RVs, Spitzer and MOST}

We performed several global Bayesian analyses with the adaptative MCMC algorithm presented in [6], using as input data our Spitzer transit photometry (see [4] for the data reduction procedure), but also the detrended MOST transit light curves and the 171 published Keck-HIRES RVs [1][2]. Analyzing the Spitzer data alone, we found that the Spitzer detected transit fully confirms, within $1-\sigma$, the ephemeris provided by [2] (Fig. 1).

Analyzing together the Spitzer photometry and the Keck-HIRES RVs, our MCMC simulation gives the median values and $68.3 \%$ probability interval for the jump parameters, as well as the derived stellar and planetary physical parameters, shown in Table 1. Our MCMC results give a planetary radius of $R_{P}=2.247_{-0.095}^{+0.098} R_{\oplus}$ as measured in IRAC $4.5 \mu \mathrm{m}$ channel, and a planetary mass of $M_{P}=7.55_{-0.79}^{+0.83} M_{\oplus}$. This yields a super-Earth with an intermediate average density $\left(\rho_{P}=3.90_{-0.61}^{+0.70} \mathrm{~g} \mathrm{~cm}^{-3}\right)$.

\section{HD 97658 b, a key object for super-Earth characterization}

\subsection{Internal composition of HD 97658 b}

The average density of HD $97658 \mathrm{~b}$ is close to the average density of Mars $\left(\rho_{O^{7}}=3.9335 \mathrm{~g} \mathrm{~cm}^{-3}\right)$. [7] proposed a model for the mass-radius relationship for rocky planets as $M_{P}=a R_{P}^{4}$, where $a$ depends on the composition. For the same composition, the average density of a planet therefore increases as $\rho_{P} \sim M_{P} / R_{P}^{3} \sim M_{P}^{0.25}$. Given that HD $97658 \mathrm{~b}$ is 75 times more massive than Mars, it clearly has a much lighter composition.

Figure 2 presents a ternary diagram for HD 97658 b, computed from various theoretical internal structures using the internal structure model described in [8], suitable for rocky and gaseous planets. Ternary diagrams relate the composition in terms of Earth-like nucleus fraction, water+ices fraction, 
Table 1. Median and 1- $\sigma$ limits of the posterior distributions derived for HD 97658 and its planet from our MCMC analysis of Spitzer photometry and Keck-HIRES RVs. Only the most relevant parameters for the discussion here are included. Full table can be found in [4].

\begin{tabular}{llll}
\hline Parameter & Symbol & Value & Unit \\
\hline Jump parameter, uniform prior & & & \\
Transit depth, Spitzer & $d F$ & $773 \pm 42$ & ppm \\
Mid-transit time-2450000 & $T_{0}$ & $6523.12540_{-0.00056}^{+0.0000}$ & BJD_TDB \\
Orbital period & $P$ & $9.4903_{-0.0015}^{+0.0015}$ & days \\
Jump parameter, Gaussian prior & & & \\
Stellar effective temperature & $T_{\text {eff }}$ & $5170 \pm 50$ & $\mathrm{~K}$ \\
Stellar metallicity & {$[\mathrm{Fe} / \mathrm{H}]$} & $-0.23 \pm 0.03$ & \\
Stellar luminosity & $L_{*}$ & $0.355 \pm 0.018$ & $L_{\odot}$ \\
Stellar mass & $M_{*}$ & $0.77 \pm 0.05$ & $M_{\odot}$ \\
\hline Derived stellar parameters & & & \\
\hline Stellar radius & $R_{*}$ & $0.741_{-0.023}^{+0.024}$ & $R_{\odot}$ \\
Stellar density & $\rho_{*}$ & $1.89_{-0.20}^{+0.23}$ & $\rho_{\odot}$ \\
Stellar surface gravity & $\log g_{*}$ & $4.583_{-0.054}^{+0.047}$ & \\
Distance & $d$ & $21.11 \pm 0.34$ & $\mathrm{pc}$ \\
\hline Derived planet parameters & & & \\
\hline Planet radius (at 4.5 $\mu \mathrm{m})$ & $R_{P}$ & $2.247_{-0.095}^{+0.098}$ & $R_{\oplus}$ \\
Planet mass & $M_{P}$ & $7.55_{-0.79}^{+0.83}$ & $M_{\oplus}$ \\
Planet density & $\rho_{P}$ & $3.90_{-0.61}^{+0.70}$ & $\mathrm{~g} \mathrm{~cm}$ \\
Planet surface gravity & $\log g_{P}$ & $3.166_{-0.061}^{+0.059}$ & \\
Orbital inclination & $i$ & $89.14_{-0.36}^{+0.52}$ & $\mathrm{deg}$ \\
Planet equilibrium temperature & $T_{\mathrm{eq}}$ & $757_{-13}^{+12}$ & $\mathrm{~K}$ \\
\hline
\end{tabular}

and $\mathrm{H}-\mathrm{He}$ fraction to total mass, to the radius for a specific planetary mass. The possible compositions for HD $97658 \mathrm{~b}$ are in the low right corner of Fig. 2 (shaded in black) and correspond to a bulk composition of $\mathrm{H}-\mathrm{He}$ of less than $2 \%$, water+ices $0-40 \%$, and rocks in excess of $60 \%$. The maximum $\mathrm{H}-\mathrm{He}$ is obtained for a rock fraction of $92-95 \%$, and the rest water+ices. If the planet had no water, the amount of $\mathrm{H}-\mathrm{He}$ would be less than $8 \times 10^{-3}$ by mass. If it had no $\mathrm{H}-\mathrm{He}$, the amount of water+ices is $15-40 \%$ by mass (the range reflects the one sigma errors in mass and radius). HD $97658 \mathrm{~b}$ is a compact planet and requires a large proportion of rocks (60-99\%), thus requiring a formation mechanism that captures enough solid material.

\subsection{Improving the knowledge of the host star}

For low-mass stars such as HD 97658, the stellar age is essentially unconstrained by the comparison with evolutionary tracks. However, the age of the star is an excellent proxy for the age of its planets, since they are expected to have formed within a few million years of each other. Asteroseismology, the study of the oscillations of stars, can help on this age determination, as we investigate here. We computed the seismic properties (the large separation $\Delta v \equiv\left\langle v_{n+1, l}-v_{n, l}\right\rangle$ and the small separations $\left.\delta v \equiv v_{n, l}-v_{n-1, l+2}\right)$ of 12 selected stellar models with the Liege adiabatic pulsation code OSC. These models have various masses, metallicities, and ages, and all simultaneously respect the observational triplet $\left(T_{\text {eff }}, L_{*},[\mathrm{Fe} / \mathrm{H}]\right)$ within the associated $1-\sigma$ uncertainties. Their properties are presented in Fig. 3 , showing that an accuracy of $\sim 1 \mu \mathrm{Hz}$ on the large and small separations measurements will help to get an improved knowledge on the stellar mass and age. The PLATO mission [9] will definitely be able to measure the large and small separations with the required accuracy. PLATO should even be able to accurately measure the individual oscillation frequencies, from which a full asteroseismic analysis can be carried out, obtaining not only very accurate global parameters (stellar mass, radius, age) but also constraints on the internal physics of the star. This is in turn important to calibrate and improve the evolutionary tracks computed from stellar evolution codes. 


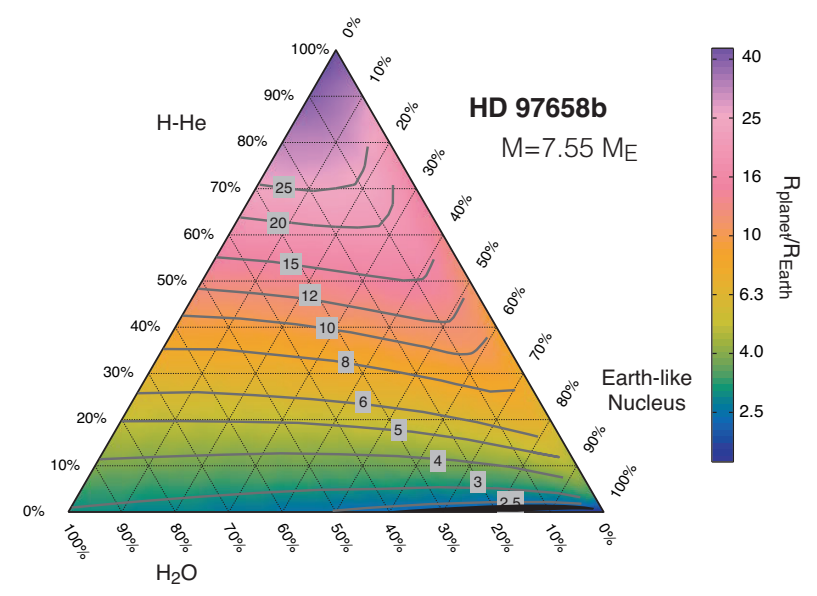

Fig. 2. Ternary Diagram for HD $97658 \mathrm{~b}\left(7.55 M_{\oplus}\right)$. Each point depicts a unique planetary composition from a combination of $\mathrm{H}-\mathrm{He}, \mathrm{H}_{2} \mathrm{O}+$ ices, and rocky earth-like nucleus (33\% iron core below a $67 \%$ silicate mantle). The color bar shows the radius in terms of Earth radii, and the gray lines are the isoradius curves labeled in terms of Earth radii. The black shaded region in the low right corner shows the possible compositions for HD $97658 \mathrm{~b}$ and the width of the shaded region takes into account the uncertainty in the mass and radius. This compact planet has at least $60 \%$ earth-like nucleus by mass and between $0-40 \%$ bulk water+ices content.

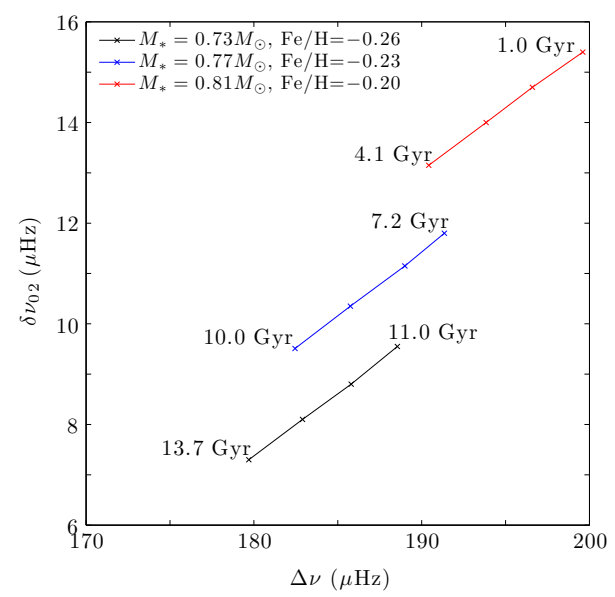

Fig. 3. Large separations $\Delta v v s$ small separations $\delta v_{02}$ for stellar models with various masses and metallicities, but that are consistent with the observational constraints $\left(T_{\text {eff }}, L_{*},[\mathrm{Fe} / \mathrm{H}]\right)$ on $\mathrm{HD} 97658$.

\section{References}

1. Howard, A., Johnson, J., Marcy, G., et al., ApJ 730, (2011) 10

2. Dragomir, D., Matthews, J., Eastman, J., et al., ApJ 772, (2013) L2

3. Gillon, M., Demory, B.-O., Deming, D., et al., IAUS 276, (2011) 167

4. Van Grootel, V., Gillon, M., Valencia, D., et al., ApJ 786, (2014) 2

5. Scuflaire, R., Théado, S., Montalban, J., et al., Ap\&SS 316, (2008) 83

6. Gillon, M., Triaud, A., Fortney, J.J., et al., A\&A 542, (2012) 4

7. Valencia, D., O'Connell, R., \& Sasselov, D. Icar 181, (2006) 545

8. Valencia, D., Guillot, T., Parmentier, V., \& Freedman, R. S., ApJ 775 (2013) 10

9. Rauer, H., Catala, C., Aerts, C., et al., ExpAst 41 (2014) 